\title{
Recent Advances in the
} Capture and Display of Macroscopic and Microscopic 3-D Scenes by Integral Imaging

\author{
This paper presents the fundamentals of integral photography and describes the \\ main contributions to its development. Attention is focused also on the recent \\ advances in both macroscopic and microscopic 3-D imaging.
}

By Manuel Martínez-Corral, Adrián Dorado, Juan Carlos Barreiro, Genaro Saavedra, and Bahram Javidi, Fellow IEEE

ABSTRACT | The capture and display of images of 3-D scenes under incoherent and polychromatic illumination is currently a hot topic of research, due to its broad applications in bioimaging, industrial procedures, military and surveillance, and even in the entertainment industry. In this context, Integral Imaging (InI) is a very competitive technology due to its capacity for recording with a single exposure the spatial-angular information of lightrays emitted by the 3-D scene. From this information, it is possible to calculate and display a collection of horizontal and vertical perspectives with high depth of field. It is also possible to calculate the irradiance of the original scene at different depths, even when these planes are partially occluded or even immersed in a scattering medium. In this paper, we describe the fundaments of InI and the main contributions to its development. We also focus our attention on the recent advances of the InI technique. Specifically, the application of Inl concept to microscopy is analyzed and the achievements in resolution and depth of field are explained. In a different context, we also present the recent advances in the capture of large scenes. The progresses in the algorithms for the calculation of displayable 3-D images and in the implementation of setups for the 3-D displays are reviewed.

Manuscript received July 19, 2016; revised January 1, 2017; accepted January 16, 2017. Date of publication March 1, 2017; date of current version April 20, 2017. This work was supported in part by the Plan Nacional I+D+I, under Grant DPI2015-66458-C2-1R, Ministerio de Economia y Competitividad, Spain, and by the Generalitat Valenciana, Spain, under Grant PROMETEOII/2014/072. The work of B. Javidi was supported under NSF/IIS-1422179 and NSF ECCS 1545687.

M. Martínez-Corral, A. Dorado, J. C. Barreiro, and G. Saavedra are with the 3D Imaging and Display Laboratory, Department of Optics, University of Valencia,

E-46100 Burjassot, Spain (e-mail: manuel.martinez@uv.es).

B. Javidi is with the Electrical and Computer Engineering Department, University of Connecticut, Storrs, CT 06269-4157 USA (e-mail: Bahram.Javidi@UConn.edu).

Digital Object Identifier: 10.1109/JPROC.2017.2655260
KEYWORDS I Computational imaging; image processing; three-dimensional imaging; three-dimensional microscopy

\section{INTRODUCTION}

Conventional photography records 2-D images of a 3-D world. This ability proceeds from the capacity of the imaging system for integrating at any pixel of the sensor all the rays that impinge on that pixel after passing through the objective lens [1]. However, if our aim is to use a photographic system to record the 3-D information of the 3-D world some modifications should be implemented to register the information about the radiance, position and direction of all the rays proceeding from the 3-D scene.

The first researcher who captured the spatial-angular information of 3-D scenes was Gabriel Lippmann, who proposed integral photography (IP) in 1908 [2]-[4]. Lippmann proposed to remove the camera lens from a photography device. Instead, he proposed to insert an array of microlenses (MLA) and a sensor (photographic film at that time) in the focal plane of the lenslets. With this system, a collection of small elemental images of the 3-D scene is recorded. Note that here we use the name integral image (InI) to refer to the collection of elemental images. The original aim of Lippmann was to use these images for building an IP monitor. Now, this original idea can be implemented by means of digital devices. This is made by displaying the InI on a pixelated display (like, for example, a tablet or a liquid-crystal computer screen), and setting a MLA just in front of the display [5]-[7]. In the past few years the improvement of features of IP monitors has 
been a matter of great interest. In this sense many research groups have made important contributions for the improvement of the depth of field [8]-[10], viewing angle [11]-[13], or display resolution [14]-[18].

More recently, the aim of capturing the spatial-angular structure of rays emitted by 3-D objects has been fulfilled with a more compact system. We refer to the system proposed by Davies and McCormick [19], and later by Adelson and Wang [20]. The technique consists of resetting a conventional camera by inserting an MLA at the image plane, and placing the sensor at the focal plane of the microlenses. For a long time this imaging system has been studied by three different, and somehow isolated, research communities, that is, the computer graphics, electrical engineers, and physical optics communities. For this reason, this device has received three different names: plenoptic camera [1], [21], [22], lightfield camera [22], [23] or far-field InI or IP camera [24], [25] while covering fundamentally the same 3-D concept and technology.

Apart from 3-D display applications, in recent years integral imaging has demonstrated great utility for the computational reconstruction of 3-D images [26]-[34]. This is very useful, for example, for the segmentation, sensing and recognition of 3-D objects [35]-[42]. Integral imaging has demonstrated recently its applicability to 3-D microscopy [43]-[46].

The aim of this contribution is to explain in a comprehensive way the theory of IP and InI, the different technical configurations for the capturing of the irradiance map from 3-D scenes, and the algorithms and technique for the depth rendering and display of the 3-D scenes. The paper is organized as follows. In Section II, we explain the concept of a radiance (or plenoptic) map of a 3-D scene. Section III is devoted to the different techniques for the capture of the plenoptic map. In Section IV, we explain and demonstrate the algorithms for the depth rendering of 3-D scenes. Section V is devoted to the display of 3-D images through IP monitors. Finally, Section VI is devoted to explaining and demonstrating the integral microscopy. In Section VII, we summarize the main outcomes of this paper.

\section{4-D RADIANCE MAP}

A ray of light can be described by the spatial coordinate $\mathbf{r}=(x, y, z)$ and the angular inclination $\theta=(\theta, \phi)$ at one point of its trajectory. In regions without scattering media and occlusions, propagation does not affect the inclination and radiance of rays. As a result, once the position and inclination of rays proceeding from a scene are known, referred to a given plane of 3-D space, one can easily compute the radiance (or plenoptic) map at any other plane. This fact permits us to reduce one dimension in the description of the plenoptic field. If we take into account that in any optical imaging system a chief propagation direction is defined, and we call $z$ such direction, we can represent the radiance map through a 4-D plenoptic function $L(\mathbf{x}, \boldsymbol{\theta})=(x, y ; \theta, \phi)$. In order to make the representations understandable, in the forthcoming graphic schemes, we will

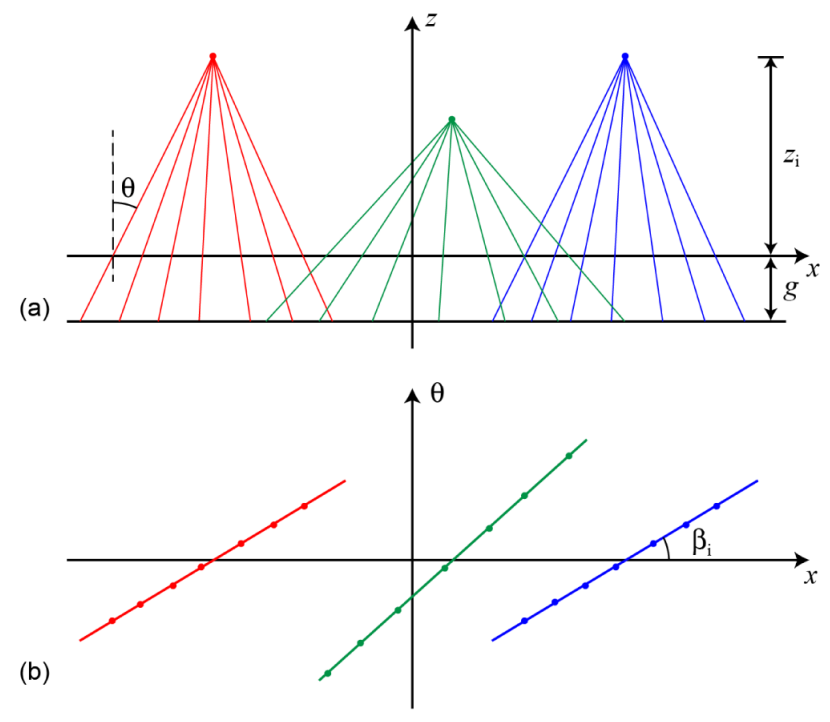

Fig. 1. (a) Scheme of ray propagation on an epipolar plane. (b) Corresponding 2-D plenoptic map.

draw an epipolar scheme. In general, an epipolar image is a 2-D slice of plenoptic function with $x+y=$ const and a zero angular value in the direction normal to this slice. However, we use a more restricted definition, so that in the forthcoming discussions an epipolar image is defined as a 2-D slice of plenoptic function, in which the value of $y$ is fixed and $\phi=0$ (or $x$ is fixed and $\theta=0$ ). To understand these concepts, in Fig. 1 we show some examples of rays propagating on an epipolar plane, and the corresponding epipolar image, which we call the 2-D plenoptic map. Specifically, in Fig. 1(a) we represent rays propagating in the $(x, z)$ plane, proceeding from emitting points set at two different distances from the abscissas axis. We have drawn also an auxiliary line at a distance $g$, to facilitate the calculation of inclination angle $\theta$. In Fig. 1(b) we show the corresponding plenoptic map. We find that any single emitting point from the 3-D scene is represented in this map by a straight segment inclined

$$
\beta_{\mathrm{i}}=\frac{1}{z_{i}} .
$$

The segment intersects the abscissas axis just at the $x$ coordinate of the emitting point.

It is apparent from the above concepts that the knowledge of the plenoptic map generated by a given 3-D scene, can permit the calculation, by means of the adequate algorithms, of the 3-D irradiance structure of the scene. In the forthcoming sections, we will review the most consolidated methods for the capture of the radiance map and the techniques for the processing and display of such 3-D information.

In order to understand the concept of epipolar image of a 3-D scene, we performed an experiment mounting a digital camera in a motorized rail. With this setup, we obtained up to 50 pictures of a 3-D scene. Between any picture and its neighbor, the camera was displaced horizontally by $3.0 \mathrm{~mm}$. 
(a)

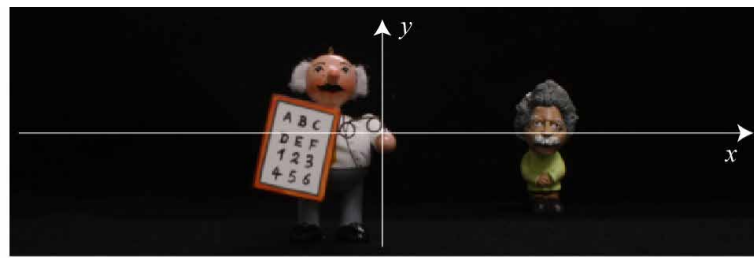

(b)

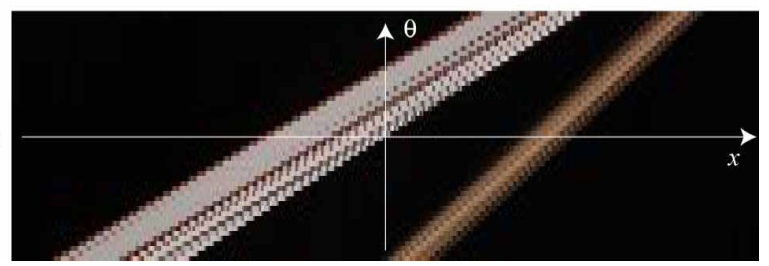

Fig. 2. (a) Central picture of the 3-D scene. (b) Epipolar image corresponding to $(y, \varphi)=(0,0)$.

The synchronized positioning, exposure, and recording of the pictures were controlled by a LabVIEW code. Fig. 2(a) shows one of the pictures (the central one) of the 3-D scene. Two objects placed at distances $30 \mathrm{~cm}$ and $45 \mathrm{~cm}$ from the camera compose the scene. In Fig. 2(b) we show the epipolar image, which was obtained by extracting and composing the central horizontal rows of the pixelated images. The computation of local slope estimates on epipolar images for the two different slice directions using the structure tensor gives two local disparity estimates for each pixel in each view. Adequate fusion of such disparity information allows the calculation of the depth map of the 3-D scene [35], [47].

\section{METHODS FOR CAPTURING THE PLENOPTIC MAP}

The first approach for the capture of the spatial-angular information of rays emitted by a 3-D scene was reported by Lippmann under the name of Integral Photography (IP). Basically, Lippmann proposed to place a microlens array (MLA) in front of a plate of photographic film, and therefore recorded a collection of elemental images of the 3-D scene. After a century passed since his original proposal, other alternative but equivalent methods have been reported in order to perform the kind of capture proposed by Lippmann. We refer, for example, to the idea of inserting an array of pinholes in front of the sensor. Note that this is a very simple method with essential limitations in light efficiency and lateral resolution. However, novel approaches based on time multiplexing are on the way for overcoming this problem [48]. Other well-known techniques are based on the idea of building an array of digital cameras [49], or the possibility of using a single digital camera on a moving platform, or obtaining multiple perspective images after subjecting the camera to an electronically controlled mechanical displacement [50]. The main advantage of later techniques is the possibility of a synthetic aperture integral imaging and controlling at will the amount of parallax and the image resolution.

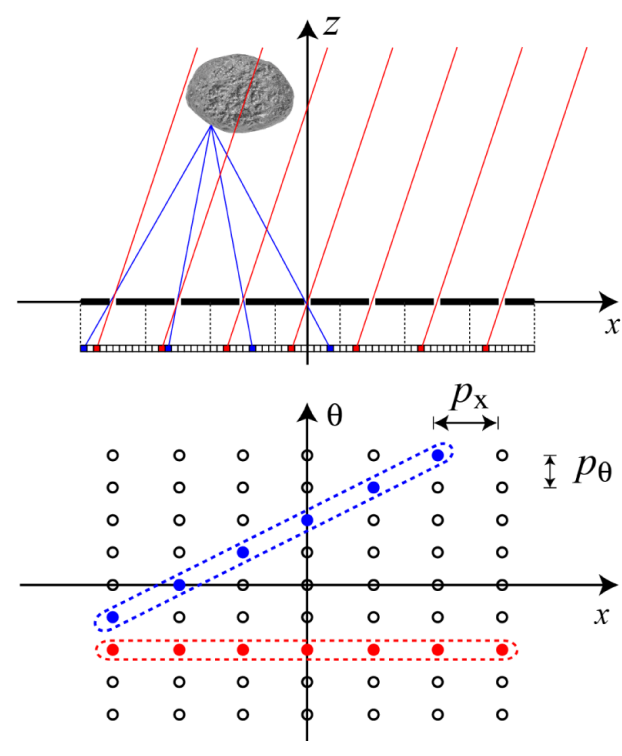

Fig. 3. (a) Scheme of system for capture of plenoptic map. (b) Corresponding 2-D plenoptic map.

Whatever the technique for capturing the integral image, it can be schematized through a very simple system in which a pinhole array is set parallel to a pixelated sensor (like a CCD). In the scheme of Fig. 3 we show a multi-imaging system in which any pinhole produces a pinhole image of the 3-D object. In order to avoid overlapping, we assume a collection of equidistant physical barriers, so that the system records an integral image composed by a collection of 2-D elemental images. This system captures a sampled version of the irradiance map. In this sense, any elemental image contains angular information of rays passing through the corresponding pinholes, and is represented by a column in the plenoptic map [see Fig. 3(b)]. It is interesting also that rays parallel to each other and passing through the pinholes are represented by a row in the plenoptic diagram. These pixels can be grouped to form a perspective (or orthographic) view of the 3-D scene.

The system schematized above is very useful when aiming to capture, with large parallax, 3-D images of large scenes; it has, however, the drawback of needing to align and synchronize an array of digital cameras.

An alternative method, but more useful when smaller parallax is acceptable, is the plenoptic camera. This new instrument is arranged by inserting an MLA at the, otherwise, sensor position of a conventional photographic camera (see Fig. 4). In this architecture, the 3-D scene is not directly in front of the MLA, but projected in its neighborhood. In order to avoid detrimental vignetting effects, the sensor is placed at the plane conjugated with the camera lens through the microlenses. To avoid overlapping between microimages the aperture angle of the camera lens must be equal to that of the microlenses. These two conditions create optical vignetting barriers between the neighboring microimages. 


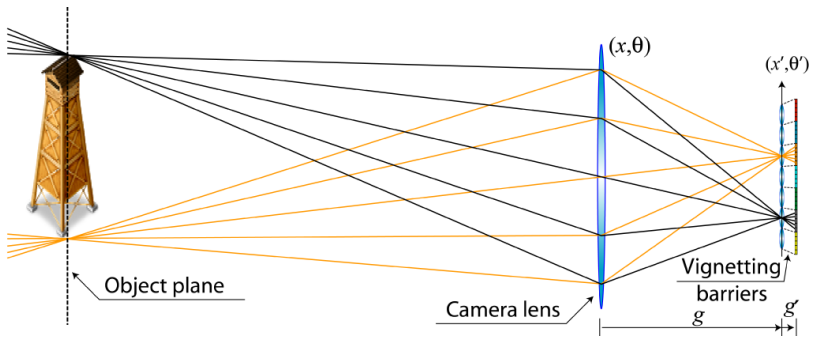

Fig. 4. Scheme of the plenoptic camera.

Next, in Fig. 5 we represent the plenoptic field recorded with the camera of Fig. 4. It is apparent that the central microimage is just behind its corresponding microlens. However, the other microimages are displaced outwards. This explains the vertical sheared form of the captured radiance map.

The outwards displacement of outer microimages can produce a significant reduction in their optical quality. This is due to the oblique incidence of optical rays, which can produce an astigmatic effect. To avoid this problem, the use of telecentric objectives has been proposed recently [51]. Although the actual design of telecentric objectives is much more complex than the scheme shown in Fig. 6, such a scheme is helpful to understand the utility of telecentricity. Note that when the exit pupil, i.e., the image of the aperture stop (in this case lens $L_{1}$ ) is at infinity, any ray passing through the center of $\mathrm{L}_{1}$ emerges from $\mathrm{L}_{2}$ parallel to the optical axis. In this case, all the microimages are set in front of the corresponding microlenses, free of outwards displacement, therefore avoiding the astigmatic effect.

In both cases, telecentric and no telecentric, the pixels in any column of the plenoptic map correspond to the microimages. Subimages are computed by grouping pixels with the same position at the microimage (see encircled points in Fig. 5).

\section{DEPTH RECONSTRUCTION OF 3-D IMAGES}

This section is devoted to the explanation of different procedures for the calculation of the irradiance distribution at planes within the 3-D scene and perpendicular to the

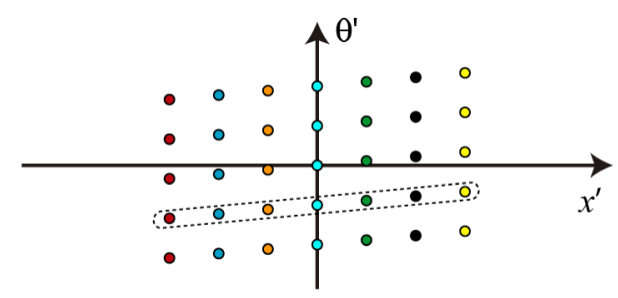

Fig. 5. The sampled plenoptic field (or radiance map) captured with the plenoptic camera of previous figure.

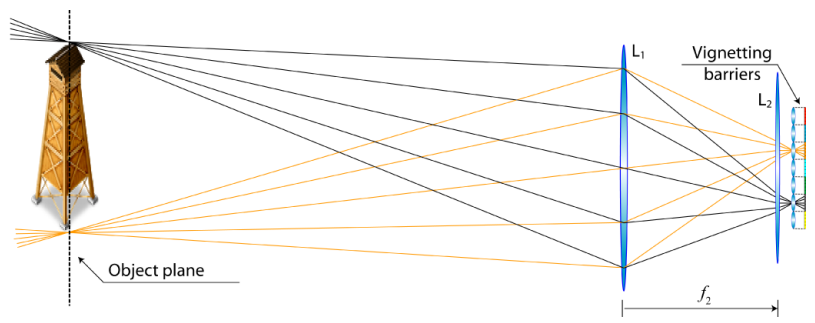

Fig. 6. Scheme of a telecentric plenoptic camera.

optical axis. This process is usually known as computational refocusing and also as 3-D reconstruction. For this task, it is necessary to describe the way the radiance map changes when rays are propagating, or when they pass through a converging, or diverging, lens. This can be made in terms of well-known ABCD matrices [52]. Propagation in free space preserves the inclination angles, but changes the spatial coordinates. Thus, in terms of ABCD, the changes suffered by the rays after propagating a distance $t$ are

$$
\left(\begin{array}{l}
x^{\prime} \\
\theta
\end{array}\right)=\left(\begin{array}{cc}
1 & -t \\
0 & 1
\end{array}\right)\left(\begin{array}{l}
x \\
\theta
\end{array}\right)=\mathrm{T}\left(\begin{array}{l}
x \\
\theta
\end{array}\right)=\left(\begin{array}{c}
x-t \theta \\
\theta
\end{array}\right) .
$$

The changes suffered by the rays after passing through a lens of focal length $f$ are

$$
\left(\begin{array}{l}
x^{\prime} \\
\theta
\end{array}\right)=\left(\begin{array}{cc}
1 & 0 \\
1 / f & 1
\end{array}\right)\left(\begin{array}{l}
x \\
\theta
\end{array}\right)=C\left(\begin{array}{l}
x \\
\theta
\end{array}\right)=\left(\begin{array}{c}
x \\
x / f+\theta
\end{array}\right) .
$$

These simple relations are very useful, for example establishing a clear relation between the two techniques described in Section III. Specifically, we can calculate the relation between the plenoptic map at the front focal plane (FFP) and the back focal plane (BFP), of a converging lens. We obtain

$$
\left(\begin{array}{l}
x_{0} \\
\theta_{0}
\end{array}\right)=(T \cdot C \cdot T)^{-1}\left(\begin{array}{l}
x^{\prime} \\
\theta^{\prime}
\end{array}\right)=\left(\begin{array}{cc}
0 & f \\
-1 / f & 0
\end{array}\right)\left(\begin{array}{l}
x^{\prime} \\
\theta^{\prime}
\end{array}\right)
$$

where $\left(x_{0}, \theta_{0}\right)$ and $\left(x^{\prime}, \theta^{\prime}\right)$ denote the spatial-angular coordinates at the FFP and the BFP, respectively.

From this equation, it is easy to see that the plenoptic map is rotated by $\pi / 2$ when propagating between the FFP and BFP of a lens. In other words, the irradiance map captured by the MLA of a plenoptic camera is the same (but rotated) as the one that could be captured by an array of lenses (or of digital cameras) placed at the FFP of the camera-lens. In the case shown in Fig. 4, in which the MLA is placed at a distance $g>f$ from the camera lens, where we consider the plane $(x, \theta)$ of the camera lens, we have an affine transformation that is the combination of shearing and rotation. In any case the effect is basically the same as the one described previously, and we can state that the map captured with the plenoptic camera is equivalent to the map that could be captured with an array of lenses placed at the plane of the camera lens (see Fig. 7). 


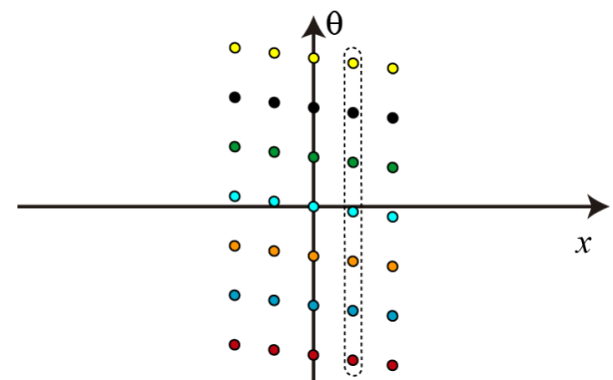

Fig. 7. Plenoptic map obtained after applying the affine transformation to the map shown in Fig. 5.

This relation relaxes the task of explaining the algorithms for the reconstruction of 3-D images. Note that, independently of the method used for the capture, the plenoptic field can be transformed in a map whose structure is equivalent to the one obtained with an array of cameras.

An interesting property of the radiance map, evaluated in a given plane $\mathbf{x}=(x, y)$, is that it is possible to calculate the irradiance distribution at such a plane by summing the radiances with the same spatial coordinate. This is made by application of the Abel transform [53] to the plenoptic map, that is

$$
I(\mathbf{x})=\int L(\mathbf{x}, \boldsymbol{\theta}) \mathrm{d} \boldsymbol{\theta}
$$

Now, from the plenoptic map captured with a system equivalent to that shown in Fig. 3 (or captured with a plenoptic camera and later rotated) it is easy to calculate irradiance distribution in planes within the 3-D scene, by simply applying the Abel transform to the propagated map, that is

$$
I(\mathbf{x}, z)=\int L(\mathbf{x}-z \boldsymbol{\theta}, \boldsymbol{\theta}) d \boldsymbol{\theta} .
$$

When integral photography is used as a typical computational imaging technique, the resolution of reconstructed images is restricted by the resolution of acquired images, but also is determined by applied algorithm. It is important when applying the algorithm to fix the number of pixels of reconstructed images. Overestimating the number of pixels results in much longer computation times, without any effective improvement of final resolution. In Fig. 8 we show an example of application of (6). In Fig. 8(a), we have drawn the result of an optimum estimation of number of pixels. In this case, the points of the plenoptic map are projected exactly onto the center of the pixels. However, in Fig. 8(b) we have considered the case of an underestimation of the number of pixels (or overestimation of pixels size). In this case, the spacing between projected points is smaller than the pixel size. This gives rise to a reduction of resolution and to an aliasing effect.

The computational depth rendering can be made by use of different algorithms. Note, however, that most of them are based on the principle of shearing the irradiance map
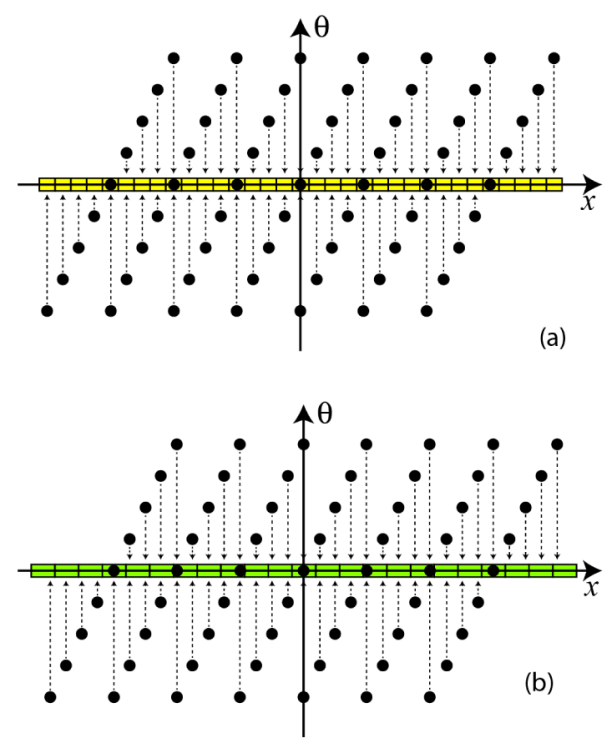

Fig. 8. Illustration of Abel transform of propagated plenoptic field. (a) Perfect matching between pixel size and projected-points spacing. (b) Overestimated pixel size.

and applying the Abel transform. This procedure can be understood as the result of projecting and summing pixels, see Fig. 9. The main problem of this method, as clearly illustrated in the figure, is that the number of pixels in the reconstructed image depends on the depth of the plane. To avoid this problem, a back-projection algorithm was reported, in which the number of pixels of reconstruction planes is fixed and is the same for every plane [18]. In this realization of back-projection algorithm, one selects first the size and number of pixels of the reconstructed image. Then, the intensity value at any pixel is obtained by summing up the values of the pixels from the elemental images that are impacted by the straight lines (or light rays) defined by the pixel from the reconstruction plane, and the center of the corresponding microlens. The main drawback of this realization is the poor efficiency in terms of computation time.

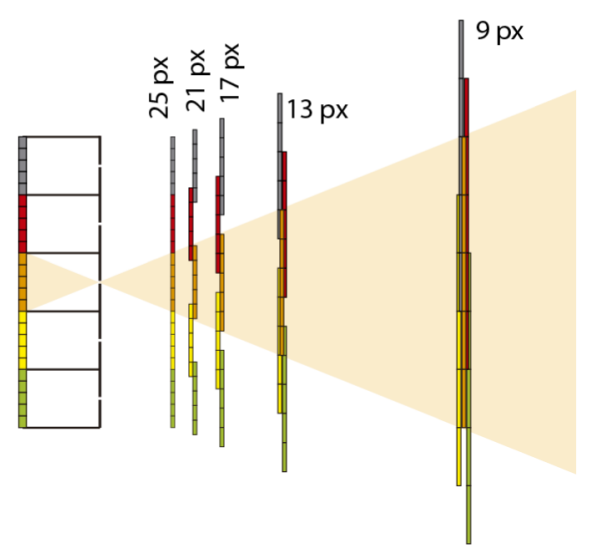

Fig. 9. Scheme for illustrating the reconstruction algorithm. 


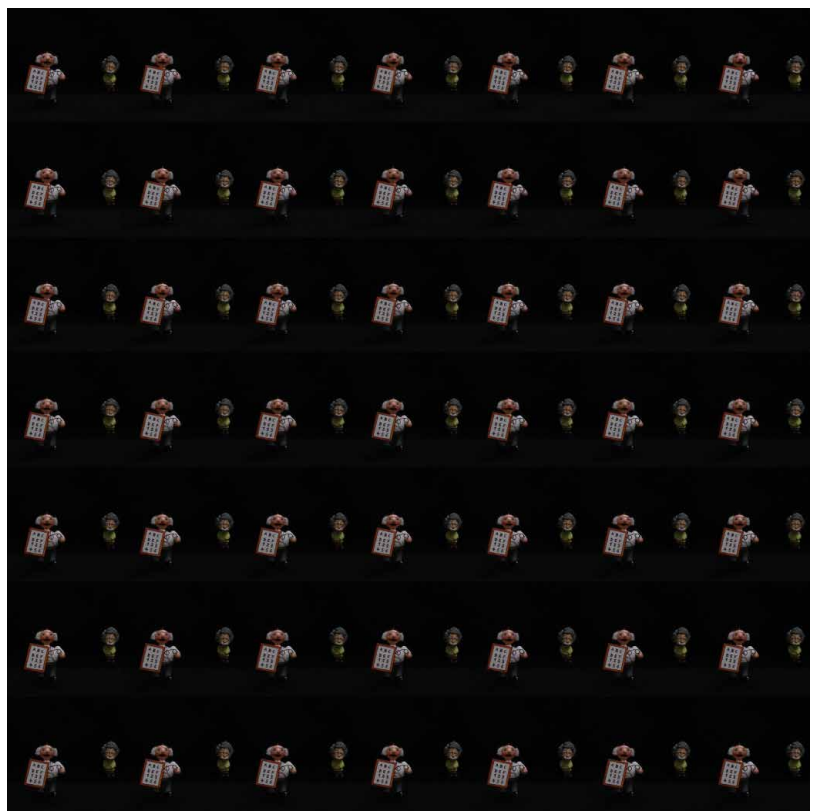

Fig. 10. Collection of $7 \times 7$ elemental images of the 3-D scene.

To illustrate the depth reconstruction procedure, we have performed an experiment with the same setup used for capturing the image shown in Fig. 2, but now recording $7 \times 7$ elemental images with pitches $p_{x}=p_{y}=5.0 \mathrm{~mm}$. The images are shown in Fig. 10. Next, in Fig. 11 we show the images reconstructed at three depths. The capacity of this computational imaging system for segmenting in depth 3-D images is apparent. This segmentation capacity has been used by some researchers for proposing methods for observation of 3-D scenes through partially occluding obstacles [54].

In recent years, some innovative methods for reconstruction have been proposed. Let us concentrate on two new methods that are both based on the quasi-periodic structure of the integral image. As shown, for example, in Fig. 10, the integral image is the periodic repetition of 2-D images of a $3-\mathrm{D}$ scene. In this case the optometrist doll, and the other doll are repeated periodically. But the two periods are different. In fact, there is an inverse proportionality between the depth position of an object and the corresponding period. In this context one of the methods is based in extracting depth images by correlating the integral image with a quasiperiodic array of single pixels [55]. Another proposal is to apply not a correlation, but a deconvolution procedure. In

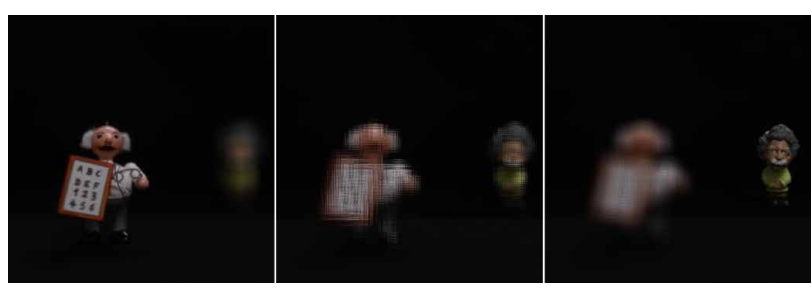

Fig. 11. Reconstructed images at three depths. this case the use of a synthetic quasi-periodic point-spread function (PSF) permits us to extract depth images [56]. The main advantage of this method is its capacity for providing high-quality reconstruction from few elemental images, and also the possibility of creating synthetic nonconventional PSFs, which permit, for example, the simultaneous segmentation of two depth planes, or the segmentation of horizontal or vertical depth structures.

\section{DISPLAY OF 3-D IMAGES}

Originally, IP technique was reported by Lippmann aiming to develop a procedure for the display of 3-D scenes. His pioneering idea was to insert the (photographically developed) array of elemental images in front of a MLA similar to the one used in the capture. When the elemental images are back illuminated, the different perspectives are integrated as a 3-D image. Every emitting point generates a cylindrical ray bundle when passing through its corresponding microlens. The intersection of many ray bundles produces a local concentration of light. The resulting scene is then perceived as 3-D by the observer. The lateral resolution of reconstructed 3-D image is determined by the size of microlenses. The amount of parallax is determined by the angular size of the MLA as seen from the center of the 3-D scene. The number of pixels behind any microlens determines the angular resolution. In Fig. 12, we show a scheme for illustrating this process.

The original Lippmann scheme has the drawback of its lack of flexibility. The experimental parameters fixed in the capture fully determine the display geometry. Despite this restriction, many proposals have been reported in the past few years taking profit from recently developed ultrahigh resolution TV screens [6], [7]. However, as described in Section III, there are other methods for the capture of the plenoptic field, which managed smartly, can display 3-D images with great flexibility.

(a)

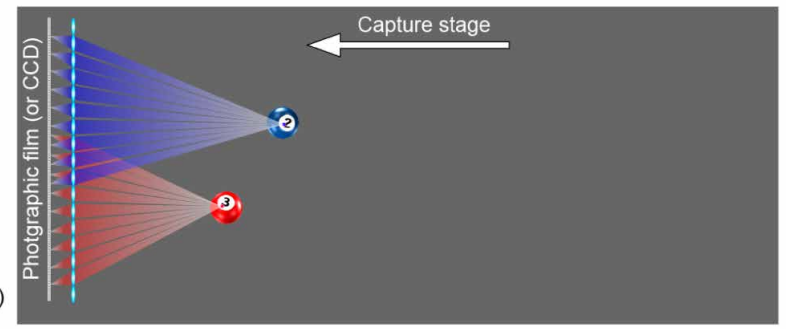

(b)

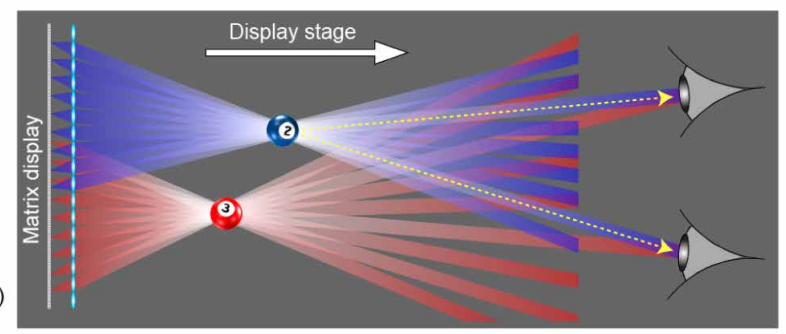

Fig. 12. Scheme of IP concept. (a) Capture stage. (b) Display stage, which produces floating 3-D images in front of the monitor. 


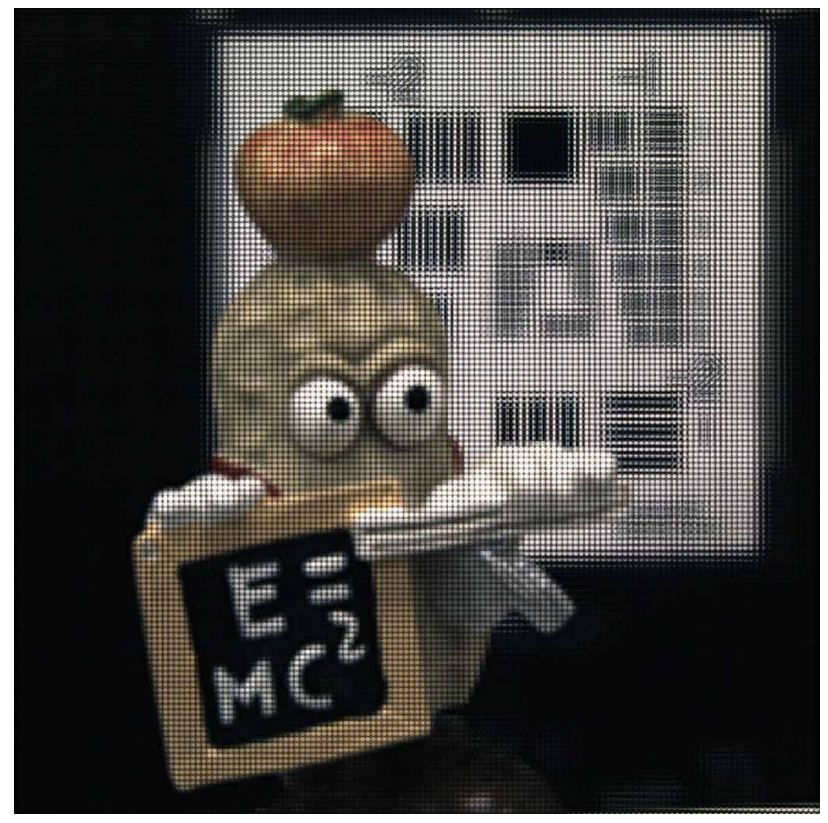

Fig. 13. Plenoptic frame of a 3-D scene.

To understand these other possibilities, let us start by remarking that: a) the IP monitor is composed of a display panel (like a LC device) and an MLA aligned in front of it; b) to allow good lateral resolution in the displayed images, the MLA should have a high number of small microlenses; c) the number of pixels behind any microlens should be high (our own experience tells us that 12 to 16 pixels per microlens are enough to produce reasonable continuity perception when changing the perspective angle); and d) the 3-D image should be displayed in the neighborhood of the display panel, with some parts floating in front of the panel and other parts behind it.

An easy solution of the aim of fulfilling all the above conditions is by displaying, on the display panel, a frame recorded with a plenoptic camera. This can be made by taking profit from some plenoptic cameras that are commercially available [57], [58], or by implementing in the laboratory a plenoptic camera in open configuration. In our case, we implemented the camera by inserting in front of the sensor of a digital camera an MLA with lenslets of focal length $f_{L}=0.93 \mathrm{~mm}$ arranged in square grid of pitch $p_{x}=p_{y}=0.222 \mathrm{~mm}$ (APO-Q-P222-F0.93 model from AMUS). With this setup, we obtained the plenoptic frame shown in Fig. 13. The parts of the frame that are apparently in focus correspond to regions close to the object plane, as defined in Fig. 4.

Then, we displayed the frame on an IP monitor composed by an iPad with retina display $(2048 \times 1536$ RGB pixels with width $\Delta_{x}=\Delta_{y}=89.0 \mu \mathrm{m}$ ). The microlens array was composed by lenses with $f_{L}=3.3 \mathrm{~mm}$ and pitch $p_{x}=$ $p_{y}=1.0 \mathrm{~mm}$. The result of the display is shown in Fig. 14 . Note that parts of the scene that are apparently defocused in the frame are reconstructed sharply in focus but behind the
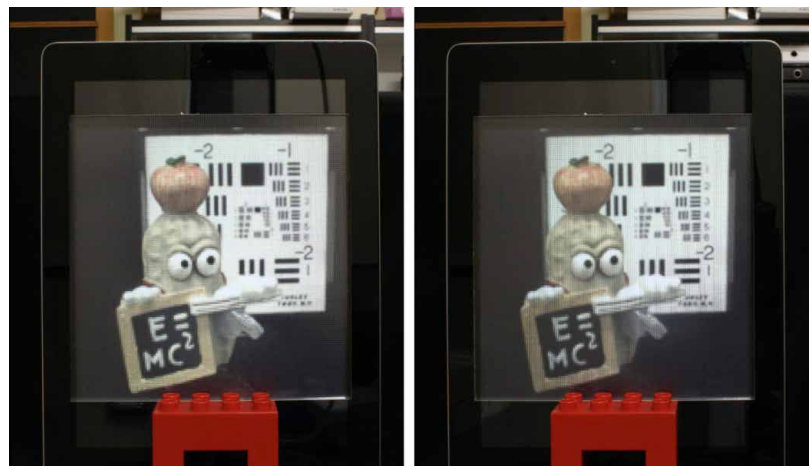

Fig. 14. Two views of the 3-D image as displayed by the IP monitor.

panel or floating in front of it. This is what we expect from this technique, since the apparent blurring in the plenoptic frame is due to the accumulation of angular information in the microimages. A limiting feature is that the parallax obtained is small. This is because in a plenoptic camera the parallax is determined by the $f$-number of the objective, but as seen from the center of the 3-D scene. Thus, in terms of 3-D display the plenoptic cameras are useful only in the case of small and close scenes.

Parallax-limitation problems do not occur in the case of IP capturing setups, in which the parallax can be set, below certain limits, at will by simply arranging a grid of digital cameras with sufficient viewing angle. However, previous to their projection onto the monitor, the elemental images captured by IP setups need to be transformed computationally. The transformation algorithm must match the number of microimages with the number of microlenses of the IP monitor and move the 3-D scene to the neighborhood of the MLA. Besides, it must reverse the pseudoscopic nature of IP images. Among the proposed algorithms [59], [60], an effective one is the so-called Smart Pseudoscopic to Orthoscopic Conversion (SPOC) algorithm [61]. The main advantage of this algorithm, which is graphically described in Fig. 15, is that it permits the computation of an integral image in which the pitch, viewing angle, number of pixels, and the number of microimages are selected at will. Other advantages are that the algorithm allows the reversion of pseudoscopy and permits us to fix the position of the object plane. The main problem of this algorithm is that outer microimages show large black zones (zero pixels), which worsen the quality of the displayed images [62]. The zero pixels are those that do not intersect with any microlens from the capturing scheme (see blue line in Fig. 15).

More recently, a novel algorithm, that takes profit from the transposition relation between IP frames and plenoptic frames, has been reported. This new algorithm takes advantage from the fact that plenoptic frames are ready to be projected onto IP monitors. In the application, it is necessary to rotate by $\pi / 2$ the spatial-angular information captured with the IP setup. But before the computational rotation some manipulations over the captured elemental images are 


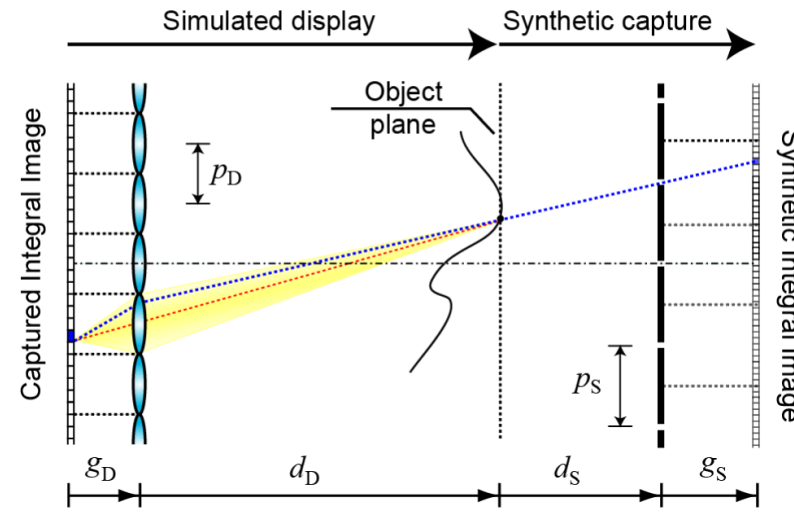

Fig. 15. Scheme of SPOC algorithm. In the simulated display the set of elemental images captured experimentally is used as the input for the algorithm. The synthetic capture is done through a simulated array of pinholes.

possible. For example, by cropping all the elemental images one can narrow their field of view, and therefore simulate an approximation of the scene. Besides, if one displaces the center of the cropping window proportionally to the distance of the elemental image to the center of the integral image, one can select the plane that is displayed at the monitor plane position. In summary, a smart cropping of the elemental images prior to the rotation permits us to select at will the object plane, the viewing angle and the lateral center of the displayed image [63], [64].

As an example, we have applied this algorithm to the elemental images shown in Fig. 10. In Fig. 16, we show the microimages calculated with the algorithm, which are ready

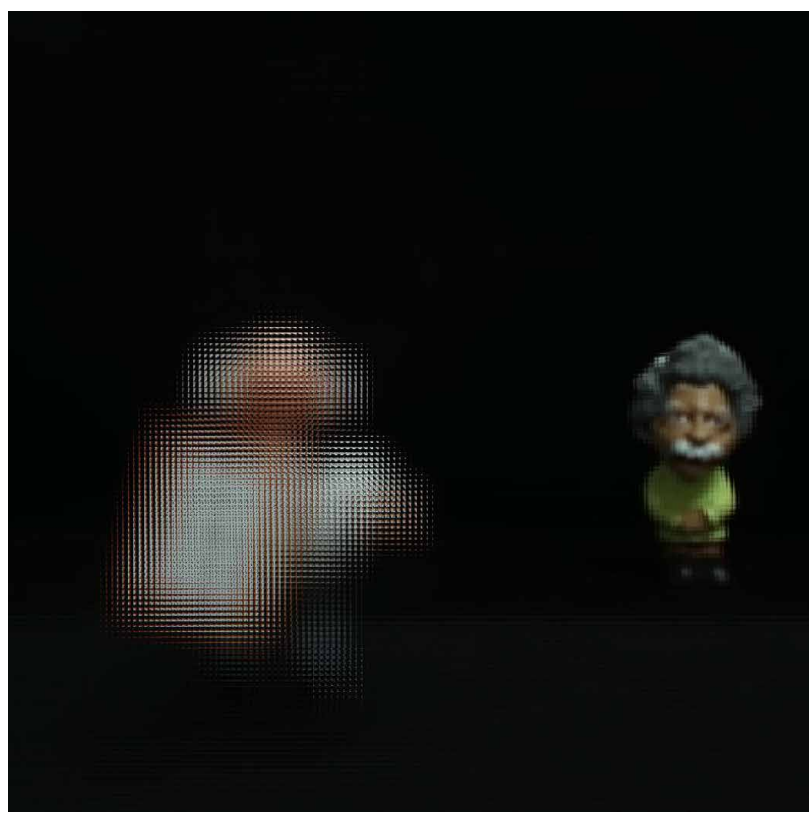

Fig. 16. Calculated microimages. For the calculation we have fixed the position of the object plane.

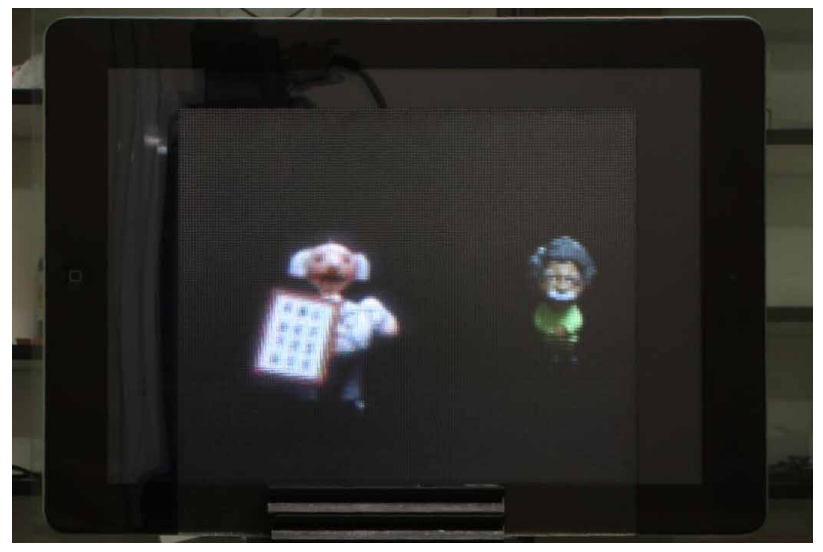

Fig. 17. Frontal view of the IP monitor.

for their projection onto the tablet. Finally, in Fig. 17 we show a view of the corresponding IP monitor.

\section{INTEGRAL MICROSCOPY}

As explained in previous sections, plenoptic cameras are especially useful for obtaining 3-D images of objects that are small and very close to the objective lens. These conditions are clearly fulfilled in microscopy. Thus, it is a natural consequence to apply IP concept to microscopy. The first proposal in this sense was made by Jang and Javidi [43], who applied this technology with the aim of displaying microscopic images. Later, in 2006, Levoy et al. proposed an integral microscope [23], and Javidi et al. proposed using an integral imaging microscope for 3-D cell identification [46].

However, the main drawback of this microscope was its poor resolution. In addition, imaging in scattering media can potentially impair the ability to visualize the scene [65]. Several attempts have been made, mainly by computational methods, to improve the lateral resolution [66]-[70]. However, no definitive improvement can be achieved if the optical part of this computational imaging technique is not improved. Let us point out that this technique has been named different terms, like lightfield microscopy, plenoptic microscopy or integral-imaging microscopy. In a more recent contribution, and aiming to acknowledge the importance of Lippmann, the instrument has been named as the integral microscope (iMic) [71].

An iMic is the result of inserting an MLA at the image plane of a conventional microscope (see Fig. 18). A conventional microscope is composed of a microscope

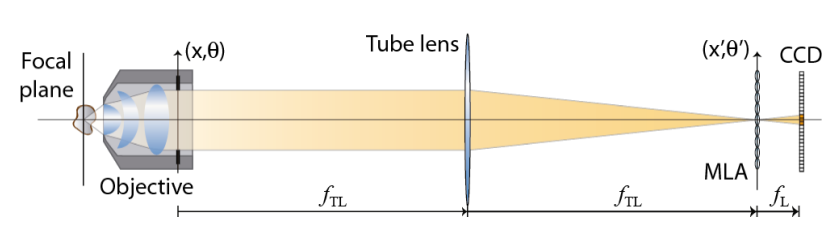

Fig. 18. Scheme of an integral microscope. 

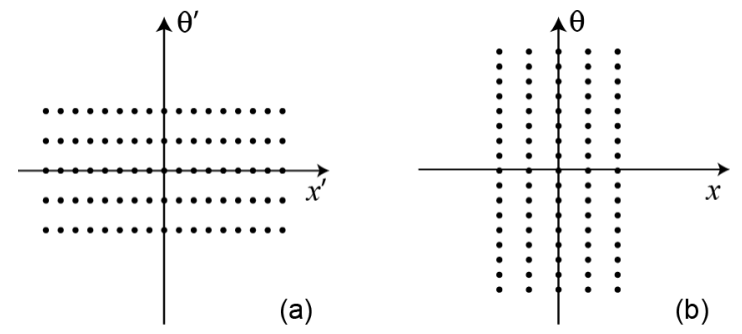

Fig. 19. Sampled plenoptic field captured with the plenoptic camera of previous figure.

objective (MO) and a tube lens (TL), which are arranged in telecentric manner. Telecentricity is a very interesting property due to, among others, two reasons: the lateral magnification does not depend on depth position of objects and any microimage is formed in front of corresponding microlens, avoiding outward shifting and degradations. To illustrate this point, in Fig. 19(a) we show a scheme of the 2-D plenoptic map captured with an iMic. In the scheme, we have taken into account that to allow images with good lateral resolution, a high number of lenslets with small pitch are required for the MLA. In contrast with the plenoptic camera (see Fig. 5), now the plenoptic map is not sheared. By simple $\pi / 2$ rotation, it is easy to calculate the plenoptic map at the aperture stop [see Fig. 19(b)]. In this map, which is not sheared either, the columns correspond to orthographic views of the 3-D object.

To show the utility of iMic, we performed an experiment by coupling a $20 x / 0.20 \mathrm{MO}$, a tube lens of $f_{\mathrm{TL}}=200 \mathrm{~mm}$, and an MLA composed by $113 \times 113$ microlenses with pitch $p=110 \mu \mathrm{m}$ and numerical aperture $N A_{L}=0.01$ (MLA \#121192-106-000 from SUSS MicroOptics). As a sample object, we used a daphnia. The microimages captured in this experiment are shown in Fig. 20. From these microimages, we calculated the perspective views, which are shown in Fig. 21.

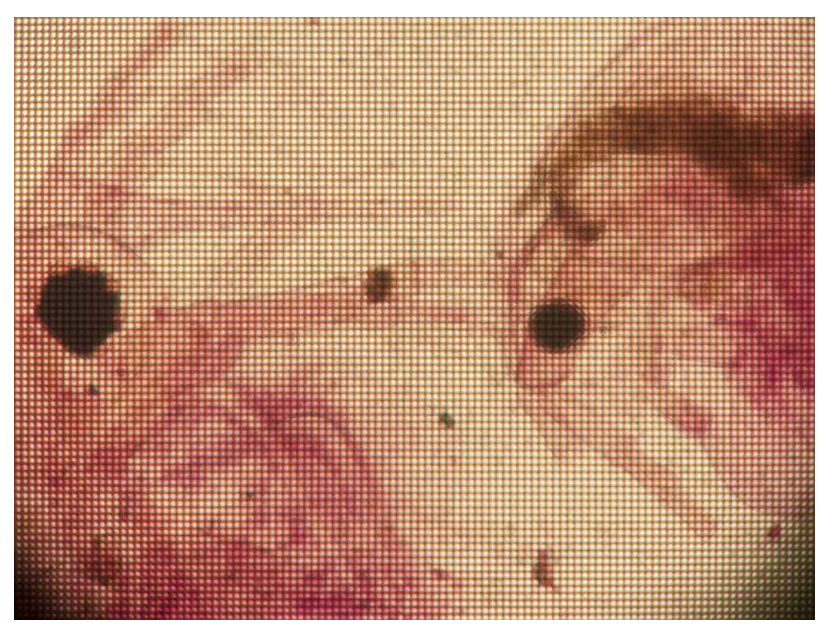

Fig. 20. Microimages recorded with the iMic.

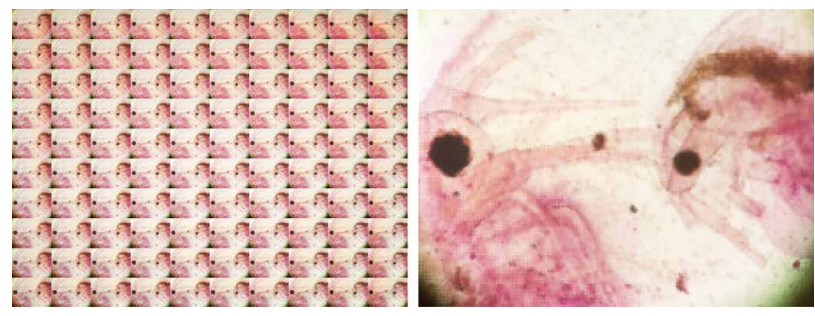

Fig. 21. Set of perspective views calculated from the microimages (left). In the right side, we show the enlarged central view.

Using the perspective views as the input of reconstruction algorithm, as described in Section IV, it is possible to obtain a reconstruction in depth of the 3-D sample. As an example, in Fig. 22 we show the irradiance distribution at one transverse plane. Note that in this reconstruction many parts of the object appear blurred.

As explained earlier, the main drawback of iMic is its poor lateral resolution. And this is a fundamental limitation since the plenoptic technique inherently involves the effective fragmentation of the aperture stop of the microscope. This implies that even in the ideal case working with perfect elements and diffraction limited imaging free of aberrations, the lateral resolution would be reduced by factor about 2.5. In the case of working with real elements, apart from diffraction there are other limiting factors, like the limiting number of pixels in the sensor, the number and size of microlenses, or the vignetting effects. In this sense, two recent initiatives for improving the resolution by optical means are remarkable. One is based on implementing a double shot system, in which two collections of microimages

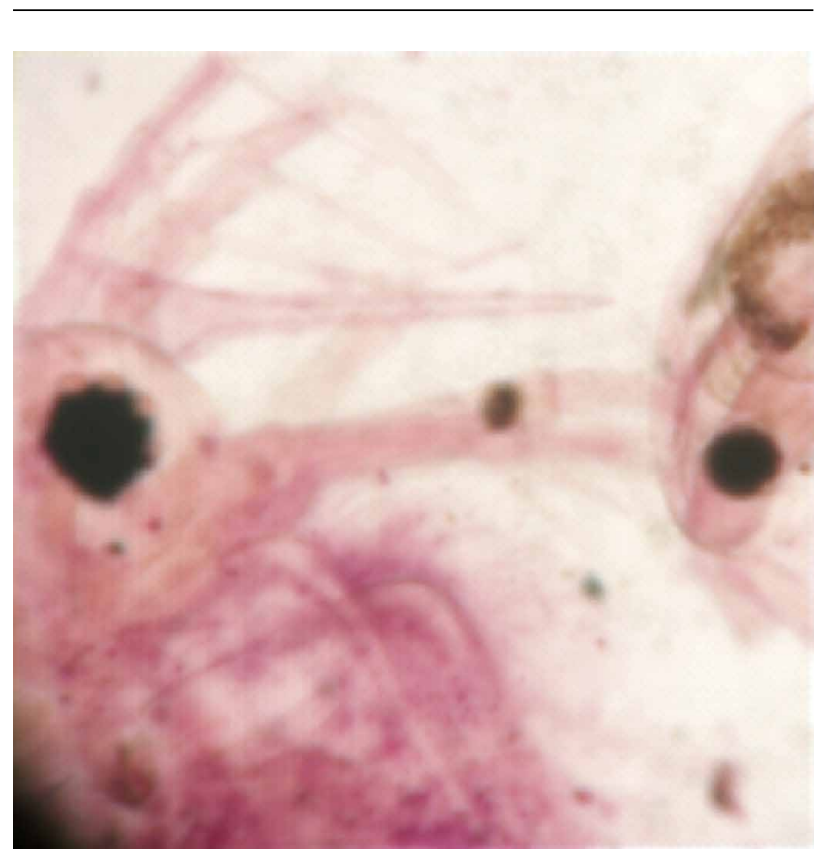

Fig. 22. Reconstructed image at one transverse plane. 
are obtained and interlaced. This has permitted overcoming the problem of limitation in size of the microlenses [71]. The other is to implement a new model of capture in which an array of digital micro-cameras is inserted at the aperture stop. This novel system has the additional advantage of enlarging the depth of field of the iMic and homogenizing in depth the lateral resolution [72], [73].

\section{CONCLUSION}

Integral photography is a technique that, although reported more than a century ago, has been revealed as being very effective for the capture, processing, and display of 3-D images of the 3-D world. In this paper, we have explained in comprehensive way the fundaments of the technique and the recent advances. We have demonstrated that integral photography offers solutions adapted to the dimensions of the 3-D objects and provides results that are increasingly competitive with other 3-D approaches.

\section{Acknowledgements}

The authors gratefully acknowledge A. Llavador (University of Valencia) for the capture of the iMic images.

\section{REFERENCES}

[1] R. Ng, "Digital light field photography," Ph.D. dissertation, Stanford Univ., Stanford, CA, USA, 2006.

[2] G. Lippmann, "Épreuves réversibles donnant la sensation du relief," J. Phys. Theory Appl., vol. 7, no. 1, pp. 821-825, 1908.

[3] H. E. Ives, "Optical properties of a Lippmann lenticulated sheet," J. Opt. Soc. Amer., vol. 21, no. 3, pp. 171-173, 1931.

[4] C. B. Burckhardt, "Optimum parameters and resolution limitation of integral photography," J. Opt. Soc. Amer. A, vol. 58, no. 1, pp. 71-74, 1968.

[5] M. Martínez-Corral, H. Navarro, R. Martínez-Cuenca, G. Saavedra, and B. Javidi, "Full parallax 3-D TV with programmable display parameters," Opt. Photon. News, vol. 22, no. 12, p. 50, 2011.

[6] J. Arai et al., "Integral three-dimensional television using a 33-megapixel imaging system," J. Display Technol., vol. 6, no. 10, pp. 422-430, 2010.

[7] M. Miura, J. Arai, T. Mishina, M. Okui, and F. Okano, "Integral imaging system with enlarged horizontal viewing angle," Proc. SPIE, vol. 8384, p. 83840, May 2012.

[8] L. Zhang, Y. Yang, X. Zhao, Z. Fang, and $\mathrm{X}$. Yuan, "Enhancement of depth-of-field in a direct projection-type integral imaging system by a negative lens array," Opt. Exp., vol. 20, no. 23, pp. 26021-26026, 2012.

[9] S. Bagheri, Z. Kavehvash, K. Mehrany, and B. Javidi, "A fast optimization method for extension of depth-of-field in threedimensional task-specific imaging systems," J. Display Technol., vol. 6, no. 10, pp. 412-421, Oct. 2010.

[10] A. Tolosa, R. Martínez-Cuenca, A. Pons, G. Saavedra, M. Martínez-Corral, and B. Javidi, "Optical implementation of microzoom arrays for parallel focusing in integral imaging," J. Opt. Soc. Amer. A, vol. 27, pp. 495-500, Mar. 2010.

[11] B. Lee, S. Jung, and J.-H. Park, "Viewingangle-enhanced integral imaging by lens switching," Opt. Lett., vol. 27, no. 10, pp. 818-820, 2002.

[12] H. Choi, S.-W. Min, S. Jung, J.-H. Park, and B. Lee, "Multiple-viewing-zone integral imaging using a dynamic barrier array for three-dimensional displays," Opt. Exp., vol. 11, no. 8, pp. 927-932, 2003.

[13] R. Martínez-Cuenca, H. Navarro, G. Saavedra, B. Javidi, and M. MartínezCorral, "Enhanced viewing-angle integral imaging by multiple-axis telecentric relay system," Opt. Exp., vol. 15, no. 24, pp. 16255-16260, 2007.

[14] J.-H. Park, K. Hong, and B. Lee, "Recent progress in three-dimensional information processing based on integral imaging," Appl. Opt., vol. 48, no. 34, pp. H77-H94, 2009.

[15] J. Arai et al., "Integral three-dimensional television with video system using pixeloffset method," Opt. Exp., vol. 21, no. 3, pp. 3474-3485, 2013.

[16] B. Javidi and J.-S. Jang, "Improved-resolution 3-D TV, video, and imaging using moving micro-optics array lens techniques and systems (MALTS)," Proc. SPIE, vol. 4902, pp. 1-12, Oct. 2002.

[17] Y.-T. Lim, J.-H. Park, K.-C. Kwon, and N. Kim, "Resolution-enhanced integral imaging microscopy that uses lens array shifting," Opt. Exp., vol. 17, no. 21, pp. 19253-19263, 2009.

[18] H. Navarro et al., "Method to remedy image degradations due to facet braiding in 3D integral-imaging monitors," J. Display Technol., vol. 6, no. 10, pp. 404-411, Oct. 2010.

[19] N. Davies, M. McCormick, and L. Yang, "Three-dimensional imaging systems: A new development," Appl. Opt., vol. 27, no. 21, pp. 4520-4528, 1988.

[20] E. H. Adelson and J. Y. A. Wang, "Single lens stereo with a plenoptic camera," IEEE Trans. Pattern Anal. Mach. Intell., vol. 14, no. 2, pp. 99-106, Feb. 1992.

[21] T. Georgiev and A. Lumsdaine, "Focused plenoptic camera and rendering," J. Electron. Imag., vol. 19, no. 2, p. 021106, 2010.

[22] R. Ng, M. Levoy, M. Brédif, G. Duval, M. Horowitz, and P. Hanrahan, "Light field photography with a hand-held plenoptic camera," Tech. Rep. CSTR 2, 2005.

[23] M. Levoy, R. Ng, A. Adams, M. Footer, and M. Horowitz, "Light field microscopy," in Proc. ACM SIGGRAPH, 2006, pp. 924-934.

[24] H. Navarro, J. C. Barreiro, G. Saavedra, M. Martínez-Corral, and B. Javidi, "Highresolution far-field integral-imaging camera by double snapshot," Opt. Exp., vol. 20, no. 2, pp. 890-895, 2012.

[25] F. Okano, J. Arai, H. Hoshino, and I. Yuyama, "Three-dimensional video system based on integral photography," Opt. Eng. vol. 38, no. 8, pp. 1072-1077, 1999.

[26] X. Xiao, B. Javidi, M. Martínez-Corral, and A. Stern, "Advances in three-dimensional integral imaging: Sensing, display, and applications," Appl. Opt., vol. 52, no. 4, pp. 546-560, 2013.
[27] L. McMillan and G. Bishop, "Plenoptic modeling: An image-based rendering system," in Proc. ACM SIGGRAPH Conf. Comp. Graph., 1995, pp. 39-46.

[28] J.-X. Chai, X. Tong, S.-C. Chan, and H.-Y. Shum, "Plenoptic sampling," in Proc. ACM SIGGRAPH Conf. Comput. Graph., 2000, pp. 307-318.

[29] S. Kishk and B. Javidi, "Improved resolution 3D object sensing and recognition using time multiplexed computational integral imaging," Opt. Exp., vol. 11, no. 26, pp. 3528-3541, 2003.

[30] S. H. Hong, J. S. Jang, and B. Javidi, "Threedimensional volumetric object reconstruction using computational integral imaging," Opt. Exp., vol. 12, no. 3, pp. 483-491, 2004.

[31] M. Levoy, "Light fields and computational imaging," Computer, vol. 39, no. 8, pp. 4655, Aug. 2006.

[32] M. Cho and B. Javidi, "Computational reconstruction of three-dimensional integral imaging by rearrangement of elemental image pixels," J. Display Technol., vol. 5, no. 2, pp. 61-65, Feb. 2009.

[33] H. Navarro et al., "Non-homogeneity of lateral resolution in integral imaging," J. Display Technol., vol. 9, no. 1, pp. 37-43, Jan. 2013.

[34] H. Arimoto and B. Javidi, "Integral threedimensional imaging with digital reconstruction," Opt. Lett., vol. 26, no. 3, pp. 157-159, 2001.

[35] M. Daneshpanah and B. Javidi, "Profilometry and optical slicing by passive three-dimensional imaging," Opt. Lett., vol. 34, no. 7, pp. 1105-1107, 2009.

[36] G. Saavedra, R. Martínez-Cuenca, M. Martínez-Corral, H. Navarro, M. Daneshpanah, and B. Javidi, "Digital slicing of 3D scenes by Fourier filtering of integral images," Opt. Exp., vol. 16, no. 22, pp. 17154-17160, 2008.

[37] J. H. Park and K. M. Jeong, "Frequency domain depth filtering of integral imaging," Opt. Exp., vol. 19, no. 19, pp. 18729-18741, 2011

[38] Y. Frauel and B. Javidi, "Digital threedimensional image correlation by use of computer-reconstructed integral imaging," Appl. Opt., vol. 41, no. 26, pp. 5488-5496, 2002.

[39] O. Matoba, E. Tajahuerce, and B. Javidi, "Real-time three-dimensional object recognition with multiple perspectives imaging," Appl. Opt., vol. 40, no. 20, pp. 3318-3325, 2001. 
[40] M. Cho and B. Javidi, "Three-dimensional visualization of objects in turbid water using integral imaging,"J. Display Technol., vol. 6, no. 10, pp. 544-547, Oct. 2010.

[41] S. H. Hong and B. Javidi, "Distortiontolerant 3D recognition of occluded objects using computational integral imaging," Opt. Exp., vol. 14, no. 25, pp. 12085-12095, 2006.

[42] M. Daneshpanah, B. Javidi, and E. A. Watson, "Three dimensional object recognition with photon counting imagery in the presence of noise," Opt. Exp., vol. 18, no. 25, pp. 26450-26460, 2010.

[43] J.-S. Jang and B. Javidi, "Three-dimensional integral imaging of micro-objects," Opt. Lett., vol. 29, no. 11, pp. 1230-1232, 2003.

[44] M. Levoy, Z. Zhang, and I. McDowall, "Recording and controlling the 4D light field in a microscope using microlens arrays," J. Microscopy, vol. 235, no. 2, pp. 144-162, 2009.

[45] Y.-T. Lim, J.-H. Park, K.-C. Kwon, and N. Kim, "Resolution-enhanced integral imaging microscopy that uses lens array shifting," Opt. Exp., vol. 17, no. 21, pp. 19253-19263, 2009.

[46] B. Javidi, I. Moon, and S. Yeom, "Threedimensional identification of biological microorganism using integral imaging," Opt. Exp., vol. 14, no. 25, pp. 12095-12107, 2006.

[47] S. Wanner and B. Goldlueke, "Variational light field analysis for disparity estimation and super-resolution," IEEE Trans. Pattern Anal. Mach. Intell., vol. 36, no. 3, pp. 606-619, Mar. 2014.

[48] A. Schwarz, J. Wang, A. Shemer, Z. Zalevsky, and B. Javidi, "Lensless three-dimensional integral imaging using variable and time multiplexed pinhole array," Opt. Lett., vol. 40, no. 8, pp. 1814-1817, 2015.

[49] B. Wilburn et al., "High performance imaging using large camera arrays," $A C M$ Trans. Graph., vol. 24, no. 3, pp. 765-776, Jul. 2005.

[50] J. S. Jang and B. Javidi, "Three-dimensional synthetic aperture integral imaging," Opt. Lett., vol. 27, no. 13, pp. 1144-1146, 2002.

[51] V. Drazic, J. J. Sacré, A. Schubert, J. Bertrand, and E. Blondé, "Optimal design and critical analysis of a high resolution video plenoptic demonstrator," J. Electron. Imag., vol. 21, p. 01007, Feb. 2012.

[52] B. E. A. Saleh and M. C. Teich, Fundamental of Photonics. Hoboken, NJ, USA: Wiley, 1991

[53] R. Gorenflo and S. Vessella, Abel Integral Equations (Lecture Notes in Mathematics), vol. 1461. New York, NY, USA: Springer, 1991.

[54] S.-H. Hong and B. Javidi, "Threedimensional visualization of partially occluded objects using integral imaging," J. Display Technol., vol. 1, no. 2, pp. 354-359, Dec. 2005.

[55] J.-Y. Jang, D.-H. Shin, and E.-S. Kim, "Optical three-dimensional refocusing from elemental images based on a sifting property of the periodic d-function array in integral-imaging," Opt. Exp., vol. 22, no. 2, pp. 1533-1550, 2014.

[56] A. Llavador, E. Sánchez-Ortiga, G. Saavedra, B. Javidi, and M. Martínez-Corral, "Freedepths reconstruction with synthetic impulse response in integral imaging," Opt. Exp., vol. 23, no. 2, pp. 30127-30135, 2015.

[57] 3D Lightfield Camera. [Online]. Available: http://www.raytrix.de/

[58] Lightfield Based Commercial Digital Still Camera. [Online]. Available: http://www. lytro.com

[59] J. Wang, X. Xiao, H. Hua, and B. Javidi, "Augmented reality 3D displays with micro integral imaging," J. Display Technol., vol. 11, no. 11, pp. 889-893, Nov. 2015.

[60] J.-H. Jung, J. Kim, and B. Lee, "Solution of pseudoscopic problem in integral imaging for real-time processing," Opt. Lett., vol. 38, no. 1, pp. 76-78, 2013.

[61] H. Navarro, R. Martínez-Cuenca, G. Saavedra, M. Martínez-Corral, and B. Javidi, "3D integral imaging display by smart pseudoscopic-to-orthoscopic conversion," Opt. Exp., vol. 18, no. 25, pp. 25573-25583, 2010 .

[62] Z. Jianlei, L. Yiqun, and W. Xiaorui, "Method for eliminating the black zone of integral imaging," in Proc. PGCIC, Nov. 2016, pp. 458-461.

[63] M. Martínez-Corral, A. Dorado, H. Navarro, G. Saavedra, and B. Javidi, "Three-dimensional display by smart pseudo-scopic-to-orthoscopic conversion with tunable focus," Appl. Opt., vol. 22, no. 5, pp. E19-E25, 2014.

[64] A. Dorado, M. Martínez-Corral, G Saavedra, and S. Hong, "Computation and display of 3D movie from a single integral photography," J. Display Technol., vol. 12, no. 7, pp. 695-700, Jul. 2016.

[65] M. Cho and B. Javidi, "Peplography-a passive 3 D photon counting imaging through scattering media," Opt. Lett., vol. 41, no. 22, pp. 5401-5404, 2016.

[66] J. Kim, J. Jung, Y. Jeong, K. Hong, and B. Lee, "Real-time integral imaging system for light field microscopy," Opt. Exp., vol. 22, no. 9, pp. 10210-10220, 2014.

[67] Y. T. Lim, J. H. Park, K. C. Kwon, and N. Kim, "Resolution-enhanced integral imaging microscopy that uses lens array shifting," Opt. Exp., vol. 17, no. 21, pp. 19252-19263, 2009.

[68] K.-C. Kwon, J.-S. Jeong, M.-U. Erdenebat, Y.-L. Piao, K.-H. Yoo, and N. Kim, "Resolution-enhancement for an orthographic-view image display in an integral imaging microscope system," Biomed. Opt. Exp., vol. 6, no. 3, pp. 736-746, 2015.

[69] M. Broxton et al., "Wave optics theory and 3-D deconvolution for the light field microscope," Opt. Exp., vol. 21, no. 21, pp. 25418-25439, 2013.

[70] N. Cohen et al., "Enhancing the performance of the light field microscope using wavefront coding," Opt. Exp., vol. 22, no. 20, pp. 24817-24839, 2014.

[71] A. Llavador, E. Sánchez-Ortiga, J. C. Barreiro, G. Saavedra, and M. Martínez-Corral, "Resolution enhancement in integral microscopy by physical interpolation," Biomed. Opt. Exp., vol. 6, no. 8, pp. 2854-2863, 2015.

[72] M. Martínez-Corral, G. Saavedra, E. Sánchez-Ortiga, A. Llavador, and J. SolaPikabea, "Microscopio integral, usos del mismo y sistema de microscopía integral," Spanish patent P201531938.

[73] A. Llavador, J. Sola-Picabea, G. Saavedra, B. Javidi, and M. Martínez-Corral, "Resolution improvements in integra microscopy with Fourier plane recording," Opt. Exp., vol. 24, no. 18, pp. 20792-20798, 2016.

\section{ABOUT THE AUTHORS}

Manuel Martínez-Corral received the Ph. D. degree in physics (Best Thesis Award) from the University of Valencia, Spain, in 1993.

Currently a Full Professor of optics at the University of Valencia, he co-leads the "3D Imaging and Display Laboratory". His research interests include resolution procedures in 3D scanning microscopy, and 3D imaging and display technologies. He has supervised $15 \mathrm{Ph}$. D. theses (three honored with the Best Thesis Award), and has published over 100 technical articles in major journals (which received more than 2000 citations).

Dr. Martinez-Corral has been a Fellow of the SPIE since 2010 and of the OSA since 2017. He has been the presenter of a number of invited and keynote presentations in international meetings. He is co-chair of

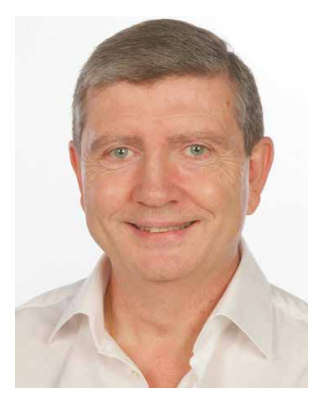

the 3D Imaging, Visualization, and Display Conference within the SPIE meeting in Defense, Security, and Sensin, as well as Topical Editor of the IEEE/OSA Journal of Display Technology and of the OSA journal Applied Optics.

Adrián Dorado was born in Spain in 1988. He received the B.Sc. and M.Sc. degrees in physics from the University of Valencia, Spain, in 2011 and 2012, respectively.

Since 2010, he has been with the 3D Imaging and Display Laboratory, Optics Department, University of Valencia. His research interests include 3D imaging acquisition and display.

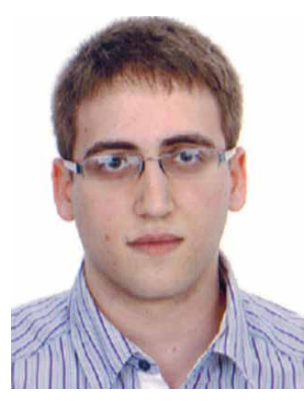


Juan Carlos Barreiro received the B.Sc. (honored with an Extraordinary Award) and Ph.D. degrees in physics from the Universitat de València, Valencia, Spain, in 1985 and 1992, respectively.

He is currently an Associate Professor in the Department of Optics, Universitat de València. In addition, he has carried out research at CIEMAT, Madrid, Spain, and as a Postdoctoral Research Fellow at INAOE, Puebla, Mexico, and at The Institute of Optics, University of Rochester, Rochester, NY, USA. Hiscurrentresearch interestsincludeoptical diffraction, high-resolution optical microscopy, and integral imaging. Besides that, he is actively involved in promoting Physics and Optics Education through outreach activities.

Genaro Saavedra received the M.Sc. and Ph.D. (cum laude) degrees in physics from the University of Valencia, Spain, in 1990 and 1996, respectively.

$\mathrm{He}$ is currently a Full Professor at the University of Valencia. Since 1999, he has been the co-leader of the 3D Imaging and Display Laboratory at that University. His research interests are optical diffraction, phase-space representation of scalar optical fields, and 3D optical microscopy. He has supervised on these

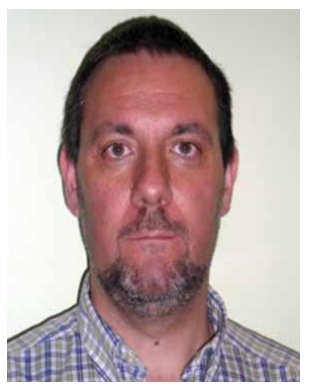
topics five Ph.D. theses (two of them honored with the Best Thesis Award). He has published about 60 technical articles on these topics in major journals. He has published in more than 60 conference proceedings, including more than 40 invited presentations. He filed seven patents on 3D display and imaging techniques.

Bahram Javidi (Fellow, IEEE) received the B.S. degree from George Washington University, USA, and the M.S. and Ph.D. degrees from the Pennsylvania State University, USA, all in electrical engineering.

He is the Board of Trustees Distinguished Professor at the University of Connecticut, Storrs, CT, USA. He has over 1000 publications, including nearly 450 peer reviewed journal articles, over 450 conference proceedings, including over 120 Plenary Addresses,

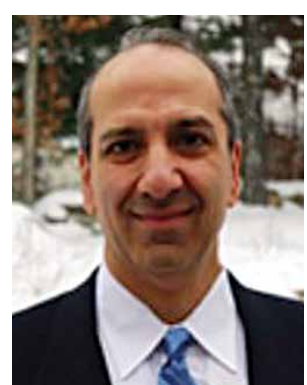

Keynote Addresses, and invited conference papers. His papers have been cited 34000 times according to the Google Scholar Citations ( $h$-index $=87$, i10-index $=537$ ). He is a co-author on nine Best Paper Awards.

Prof. Javidi received the Quantum Electronics and Optics Prize for Applied Aspects by the European Physical Society in June 2015. He has been named Fellow of several scientific societies, including IEEE, OSA, and SPIE. In 2010, he was the recipient of the George Washington University's Distinguished Alumni Scholar Award, University's highest honor for its alumni in all disciplines. In 2008, he received a Fellow award by John Simon Guggenheim Foundation. He received the 2008 IEEE Donald G. Fink prized paper award among all (over 150) IEEE Transactions, Journals, and Magazines. In 2007, The Alexander von Humboldt Foundation awarded him with the Humboldt Prize for outstanding U.S. scientists. He received the Technology Achievement Award from the SPIE in 2008. In 2005, he received the Dennis Gabor Award in Diffractive Wave Technologies from SPIE. He was the recipient of the IEEE Photonics Distinguished Lecturer Award twice in 2003/2004 and 2004/2005. He was awarded the IEEE Best Journal Paper Award from IEEE Transactions on Vehicular Technology twice in 2002 and 2005. Early in his career, the National Science Foundation named him a Presidential Young Investigator and he received The Engineering Foundation and the IEEE Faculty Initiation Award. He was selected in 2003 as one of the nation's top 160 engineers between the ages of 30 to 45 by the National Academy of Engineering (NAE) to be an invited speaker at The Frontiers of Engineering Conference which was co-sponsored by The Alexander von Humboldt Foundation. He is an alumnus of the Frontiers of Engineering of The National Academy of Engineering since 2003. He has served on the Editorial Board of the Proceedings of the IEEE Journal [ranked \#1 among all electrical engineering journals], the advisory board of the IEEE PнотоNICS JouRnAL, and he was on the founding board of editors of IEEE/OSA Journal of Display Technology. 